\title{
CORRECTION
}

\section{Correction to: "Computational Analysis of Unsteady Swirling Flow Around a Decelerating Rotating Porous Disk in Nanofluid" by Talat Rafiq and M. Mustafa, Arabian J. Sci. \& Eng. 45 (2020) 1143-1154}

Talat Rafiq ${ }^{1} \cdot$ M. Mustafa ${ }^{1}$ (D)

Published online: 24 October 2020

(c) King Fahd University of Petroleum \& Minerals 2020

\section{Correction to: \\ Arabian Journal for Science and Engineering (2020) 45:1143-1154 \\ https://doi.org/10.1007/s13369-019-04257-z}

In [1], we have assumed that suction velocity has the form $V(t)=V_{0}(1-\alpha t)^{-1}$. However, the correct form is $V(t)=$ $V_{0}(1-\alpha t)^{-1 / 2}$. This correction is necessary to obtain the condition $F(0)=A / 2$ given by Eq. (12) of [1].

Rest all the equations and the results presented in [1] are correct.

\section{Reference}

1. Rafiq, T.; Mustafa, M.: Computational analysis of unsteady swirling flow around a decelerating rotating porous disk in nanofluid. Arabian J. Sci. Eng. 45, 1143-1154 (2020)
The original article can be found online at https://doi.org/10.1007/s13 369-019-04257-z.

\section{Mustafa}

merajmustafa@sns.nust.edu.pk

1 School of Natural Sciences (SNS), National University of Sciences and Technology (NUST), Islamabad 44000, Pakistan 